\title{
A Field Theory of Entropy
}

\author{
Alireza Jamali*
}

April 24, 2021

\begin{abstract}
Starting from an argument on the impossibility of emergence of temperature within current physics, we propose to take temperature as a fundamental property of matter, with rest energy of $E=2 k_{B} T$. As an inductive consequence, this fundamental property of matter must produce a field of entropy according to the equation $\square S=-\frac{\kappa}{2} k_{B}^{2} \theta$, where $\kappa=8 \pi G / c^{4}$ is Einstein's constant, and $\theta$ temperature density per unit volume.
\end{abstract}

\section{Contents}

1 Introduction

2 On the impossibility of emergence of temperature in current system of physics

2.1 Rules of definitions in logic . . . . . . . . . . 3

2.2 Defintion of temperature . . . . . . . . . . . 4

2.3 The fundamental proposition . . . . . . . . . . 5

2.3.1 Induction on de Broglie's methodology . . . . . . . 6

3 Incompatibility of Special Relativity and Fourier's theory of Heat Conduction

4 Proposed Field Equation for Entropy $\quad 7$

4.1 Heuristic derivation of the proposed equation . . . . . . 7

4.2 Law of Conservation of Informedness . . . . . . . . . . . . . . 9

4.3 So what is heat, exactly? . . . . . . . . . . . 9

5 Regarding Blackhole Thermodynamics, Part I

*alireza.jamali.mp@gmail.com, snail-mail: 3rd Floor - Block No.6 - Akbari Alley - After Dardasht Intersection - Janbazané Sharghi - Tehran - Iran 
6 An argumentum ad absurdum

6.1 'Statistical definition' of gravitational potential and mass . 11

6.1.1 Gravitational Capacity (Massic/Specific energy) . . 11

6.1.2 Gravitational Pressure . . . . . . . . . . . . 11

6.1.3 First 'law' of Gravitodynamics . . . . . . . . . . . 11

6.2 The canonical ensemble . . . . . . . . . . . . . . . . 12

6.3 Equipartition of energy . . . . . . . . . . . . . 12

7 Mass-Charge correspondence $\quad 12$

7.1 Introduction . . . . . . . . . . . . . . . . 12

7.2 The correspondence . . . . . . . . . . . . . . . . 12

7.3 Implications . . . . . . . . . . . . . . . . . . . 13

7.3.1 Charge-Energy equivalence . . . . . . . . . . 13

7.3.2 Existence of Massless Charged particles . . . . . . . 13

7.3.3 Reissner-Nordström radius from posited Electrostatic

energy . . . . . . . . . . . . . 13

7.3.4 Schwarzschild radius from posited Gravitational en-

ergy . . . . . . . . . . . . . . . . 14

7.4 A closer look at the Reissner-Nordström metric . . . . . . . 14

7.4.1 Empirical Preference . . . . . . . . . . . . 15

8 Regarding Blackhole Thermodynamics, Part II 15

8.1 Thermal radius of a spherically symmetric entity . . . . . . 15

8.2 Inclusion of blackhole's Temperature in the metric . . . . . 16

8.2.1 Reissner-Nordström-like approach . . . . . . . 16

8.2.2 Mass-temperature correspondence approach . . . . . 16

9 Objections and Replies $\quad 16$

9.1 Temperature à la Landau-Lifshitz . . . . . . . . . . . . . . . 16

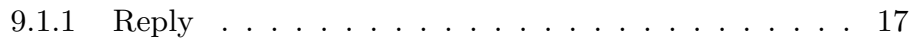

9.2 Theoretical physics methodology à la pop-science . . . . . . 18

9.2.1 Reply to: 'not systematically derived' . . . . . . . . 18

9.2.2 Reply to: 'physics is about explaining experimental observations' . . . . . . . . . . . . . 18

9.2.3 Reply to: 'does not explain anything' . . . . . . . . 19

9.2.4 Reply to: 'no observable consequences' . . . . . . . . 19

A History of evolution of author's ideas which led to field approach to Entropy

\section{Introduction}

Altogether there are two major theoretical problems with our overall understanding of heat and the related phenomena. The first is a logical problem lurking at the foundations of statistical physics which makes its goal of explanation of temperature as an emergent property of many particles impossible, and the second is the well-known Incompatibility of Special Relativity and Fourier's theory of Heat Conduction. In this paper we shall propose an equation which solves both of these problems. 


\section{On the impossibility of emergence of temperature in current system of physics}

Since understanding the logical problem requires having a clear understanding of rules of logic to avoid being deceived by fallacies, we begin by a logical analysis of definitions.

Because the purpose of a defintion is to construct a concept and to eliminate ambiguity while encompassing all the wanted examples of that concept that which we already know of, logic is what ought to be our guiding principle.

In logic, that which is being defined is called the definiendum and the words or images used to explain the meaning of the definiendum are called the definiens. For example,

A circle is a shape consisting of all points in a plane that are at a given distance from a given point.

In this example, circle is the concept that which we want to define, therefore the definiendum; And the rest of the sentence after 'is' are the definiens. Notice that this definiton of circle does indeed encompass all the intended examples of 'circle' and no more unintended ones; This is among rules that we mention in the next section.

\subsection{Rules of definitions in logic}

In order to satisfy what definitions intend to do (i.e. clarification and conceptualization), we are bound by the rules of logic on how to define a term. Besides that a defintion has to be precise and clear, a definition should not be negative where it can be affirmative and it must only state the essential attributes (not too broad not too narrow). There is a law that we are going to specially focus on, because it is most related to the subject matter of this paper: It is a logical necessity that a defintion must not be circular. Synonyms and antonyms of the definiendum or any word, concept or image which in order to define or understand it, one has to refer back to the original definiendum must be absolutely avoided and not be re-stated in the definiens, otherwise, the definition has not fulfilled its intended purpose; it will not take any advance on conceptualization of the definiendum, and it will have no theoretical value and does not help us to understand the quiddity of the definiendum or get closer to understand it.

An infinite regress can also result from successive automatic substitutions of the definiens for the definiendum located in the definiens at each step of the regress[1]. For example,

A teacher is a person who teaches.

Which is equivalent to

A teacher is a person who does what a teacher does.

Which is equivalent to

A teacher is a person who does 'what a person who does what a teacher does', does ad infinitum.

This is a tautology formally known as the fallacy of circular defintion. 


\subsection{Defintion of temperature}

According to statistical mechanics, the temperature of a system in equilibrium is defined[2][3][4] by

$$
\frac{1}{T}:=\frac{\partial S}{\partial E}
$$

where $S=k_{B} \log W(E)$. This established mathematical defintion results in other interpretations of said defintion, such as defining temperature by the average kinetic energy of particles

$$
\frac{3}{2} N k_{B} T=\frac{1}{2}\left\langle m v^{2}\right\rangle
$$

But, there is a logical problem with this defintion: Temperature is not 'the average kinetic energy of a system of many particles', it is the average kinetic energy of a system of many particles divided by the Boltzmann constant. The Boltzmann constant is not a dimensionless constant and is meaningless without its dimensions. Because the dimensions of the Boltzmann constant are an essential part of the definiens of it, and are necessary in the quiddity and what the Boltzmann constant even means, they shall not be omitted logically in statements of this constant; Here is where the logical fallacy becomes clear: In the current system of SI units, the units of the Boltzmann constant is Joules/Kelvin, but Kelvin is the base unit of measurement of temperature; this means, in order to define or to understand [temperature] (which is a necessary part of the definiens of 'temperature'), one necessarily has to refer back to the original definiendum (temperature). Therefore, this circular-dependent relation in between the definiens and the definiendum, makes this definition circular. The same is the issue with the equation (1).

From the perspective of dimensional analysis, the equation (1) of the definiens reads: [temperature]

and our definiendum reads [temperature] It is true that this is the condition of dimensional analysis: the dimensions of both sides of the equation have to be the same; but there is more to this, for this oughts to be a definition of temperature; there is not a problem at all with the dimensions being equal per se, the problem occurs when one claims this equation as a defintion for temperature, because it overlooks the logical necessity of avoidance of circular logic.

This is, however, not the case with other definitions in physics. For example, Newton's second law of motion and the defintion of 'force'

$$
\mathbf{F}:=m \mathbf{a}
$$

Here, the dimensional analysis reads

$$
\text { Newton }=k g \frac{m}{s^{2}}
$$

In which a 'Newton' is defined by $\mathrm{kgm} / \mathrm{s}^{2}$. This equation sets meaning on what [Newton] means, and no already-synonymous concept of the definiendum ('Newton') is in the definiens. 


\subsection{The fundamental proposition}

As the unit of temperature (kelvin) is one of the basic units of measurement in physics, this observation suggests that within a physics constructed upon a given set of basic units, none of the basic units can be explained to be emergent.

The logical conclusion of the above discussion is that

In the current system of physics, we propose to take temperature as a fundamental property of matter. Therefore, just like the case for mass or electric charge, a single particle can possess temperature; on the precise amount of this temperature for individual elementary particles we are still unable to say any word. But from a combination of the theorem of equipartition of energy and special relativity we can say

\begin{tabular}{|l|l|l|}
\hline Particle Type & Equipartition Energy & Associated temperature \\
\hline Massive & $E=(D / 2) N k_{B} T$ & $m c^{2}=2 k_{B} T$ \\
\hline Ultra-relativistic & $E=D N k_{B} T$ & $p c=4 k_{B} T$ \\
\hline
\end{tabular}

shall give an estimate for the rest temperature $T_{0}$ of an uncharged particle ${ }^{1}$ 2 . As we are in the realm of relativity, all dimensions must be treated equally and according to special relativity, a particle in fact moves in four dimensions, therefore in the theorem of equipartition of energy ${ }^{3}$ we have let $D=4$ and $N=1$.

An interesting instance is the rest temperature of proton,

$$
T_{0, p}=\frac{m_{0, p} c^{2}}{2 k_{B}} \approx 5.444 \times 10^{12} \text { Kelvin }
$$

which is statistically the same temperature achieved at ALICE[5].

\footnotetext{
${ }^{1}$ It is crucial to be kept in mind that the temperature of a particle is an autonomous characteristic and cannot be defined from any prior concept. Supposing that the temperature of a particle is given by

$$
T_{0}=\frac{m c^{2}}{2 k_{B}}
$$

is just like assuming that the electric charge of an electron is given by $q_{e}=\sqrt{4 \pi \epsilon_{0} G} m_{e}$, which is not true. Therefore $2 k_{B} T=m c^{2}$ must be only viewed as a motivation, which is valid under special circumstances in which, via the conservation of energy, mass is transformed into temperature and vice versa.

${ }^{2}$ Theoretical considerations alone are not sufficient in determining the numerical coefficient, i.e. whether

or

$$
E=2 k_{B} T
$$$$
E=k_{B} T
$$

This can only be decided by experiment.

${ }^{3}$ In a second reading of this paper there is a possible objection: The theorem of equipartition of energy is usually derived from the partition function, but our proposal denies any ontological basis for the statistical treatment of temperature. Our response is that the choice of the factor remains arbitrary unless a direct experimental test of the theorem of equipartition can select the proper factor; something which is extremely difficult, if not epistemologically impossible. On the other hand, it may be the case that this theorem turn out to be a principle, not derivable from prior assumptions, so we better not risk contradicting a possible principle of nature. In any case, our approach is the most consistent and safest approach that one can pursue to maintain coherency of old and new theories.
} 


\subsubsection{Induction on de Broglie's methodology}

We believe that a proposal which is not forbidden logically or empirically, must be proposed and seriously considered. Historically, this was a huge success with de Broglie's proposal. Retrospectively what de Broglie did was to use conservation of energy

$$
m c^{2}=h \nu
$$

to say that anything that can have mass must have frequency. There must exist a deeper reason why this ontological extrapolation of de Broglie worked. We call such 'deeper reason' the generalizability of energy, according to which, any property which can be attributed through a conservation of energy is ontologically justified. According to this supposed property of energy, similar to de Broglie's proposal, through

$$
m c^{2}=2 k_{B} T
$$

anything that can have mass must have temperature, and since there is no logical or empirical obstacle to assuming that temperature can be associated with a single particle, it must be assumed to be so.

Sceptics of this 'generalizability of energy' can instead appeal to induction (à la Hume, not the one in mathematics!) on what de Broglie did, to justify $T_{0}=m_{0} c^{2} / 2 k_{B}$.

\section{Incompatibility of Special Relativity and Fourier's theory of Heat Conduction}

It is well known that the Fourier equation is incompatible with the theory of relativity[6] for at least one reason: it admits infinite speed of propagation of heat signals within the continuum field. For example, consider a pulse of heat at the origin; then according to Fourier equation, it is felt (i.e. temperature changes) at any distant point, instantaneously. The speed of information propagation is faster than the speed of light in vacuum, which is inadmissible within the framework of relativity.

To proceed with overcoming this incompatibility, we must answer a question:

- What are these heat signals? In other words, what is the field of heat?

It has been long thought that the temperature scalar field $T(\mathbf{x}, t)$ is the carrier of heat signals; in which case the heat equation needs to be modified, but all such attempts of Hyperbolic heat conduction [7][8][9]

$$
\frac{1}{C^{2}} \frac{\partial^{2} T}{\partial t^{2}}+\frac{1}{\alpha} \frac{\partial T}{\partial t}=\nabla^{2} T
$$

are still unsatisfactory in that they contain both first and second derivatives, thus still not expressible in a special relativistic-invariant form. 


\section{Proposed Field Equation for Entropy}

Our fundamental proposition ${ }^{4}$ which solves both of the aforementioned problems at once, is that $S$, having dimension of entropy ${ }^{5}$, is the potential function of the heat signals and these signals propagate at the speed of light ${ }^{6}$ which is governed by the following equation

$$
\left(\frac{1}{c^{2}} \frac{\partial^{2}}{\partial t^{2}}-\nabla^{2}\right) S=\square S=-\frac{4 \pi G k_{B}^{2}}{c^{4}} \theta
$$

where $\theta$ is temperature density (per unit volume), $G$ the universal Gravitational constant, $k_{B}$ Boltzmann's constant and $c$ speed of light in vacuum ${ }^{7}$. Taken most seriously, this equation represents a new fundamental force, the third inverse-square law of physics after that of Newton and Coulomb, and the first to combine constants from the previous two. As $\Delta S=-\Delta I$ where $I$ is information[11], we absorb the minus in $S$ and take

$$
\square I=\frac{4 \pi G k_{B}^{2}}{c^{4}} \iota
$$

to be the law of information propagation in space-time. Accordingly, temperature of a particle maybe renamed as the informedness, $\iota$ of the particle. $^{8}$

In the next subsection we shall present an inductive argument for the proposed equation (2) based on the fundamental proposal that a single particle does possess temperature.

\subsection{Heuristic derivation of the proposed equation}

Two of the major characteristic properties of elementary particles, i.e. mass and electric charge, are sources for novel interactions of gravity and electricity (electromagnetism). Using induction (again not the one in mathematics) on this observation, we propose

The Principle of Property-Interaction Correspondence: To each

\footnotetext{
${ }^{4}$ The whole discussion of this paper is restricted to vacuum. We shall not deal with the analogue of 'electromagnetism in matter'.

${ }^{5}$ It need not, however, to be defined as $S=k_{B} \log W$, as with the case for BekensteinHawking entropy,

$$
S_{\mathrm{BH}}=\frac{k_{B} A}{4 l_{P}^{2}} .
$$

We shall say more on this in a forthcoming paper.

${ }^{6}$ There is not much choice about the speed of propagation when one considers a fundamental field; special relativity brute-forces all such fields to propagate at $c$, including the field of gravity (Newtonian gravitational potential).

${ }^{7}$ The (temporary) uncertainty regarding the numerical factor is also present here, i.e. $4 \pi G$ or $8 \pi G$. As will become evident by reading the heuristic derivation, we have chosen not to manipulate any equation which arises naturally. Yet, again, the ultimate judge must be experiment.

${ }^{8}$ It might be useful to distinguish $\iota$ and $\theta$ by taking $\iota$ as $-\theta$, but as $\theta$ is always positive in the absolute scale, that would mean an ever-negative $\iota$, which lacks formalistic harmony; we do not, however, deny that possibility that informedness like electric charge may be both positive and negative and it must remain open to empirical judgement.
} 
characteristic property of elementary particles there corresponds a fundamental interaction.

Therefore according to our fundamental proposal that temperature is a fundamental property of (elementary) single particles, it must as well be the source for a new force which we now proceed to find. Let us begin by the observation that if we add mass to the source term (current density) of electromagnetism, we can construct the gravitational potential and from the field equations we are led to the Poisson equation for gravity. To see this, we first construct the five-current

$$
J^{\mu}:=\left(c \sigma_{0} \mu, c \rho, \mathbf{J}\right),
$$

where $\mu$ is mass density, $\rho$ electric charge density and $\mathbf{J}$ electric current density and $\sigma_{0}:=\sqrt{4 \pi \epsilon_{0} G}$.

To construct the five-potential, we first note how the four-potential

$$
A^{\mu}:=(\varphi / c, \mathbf{A})
$$

is contructed from the four-current: divide the component of potential by the coefficient of the corresponding component of current, i.e. divide the electric potential by $c$, therefore the candidate for the gravitational component of current density would be $\phi /\left(c \sigma_{0}\right)$, where $\phi$ is the (Newtonian) gravitational potential. To validate this candidate it only remains to verify that $\phi /\left(c \sigma_{0}\right)$ has the units of Volts/c, which indeed it has. It only remains to observe that the field equation

$$
\square A^{\mu}=-\mu_{0} J^{\mu}
$$

yields

$$
\left(\frac{1}{c^{2}} \frac{\partial^{2}}{\partial t^{2}}-\nabla^{2}\right) \phi=-4 \pi G \mu
$$

assuming the field is time-indpendent, we are led to the Poisson equation for gravity

$$
\nabla^{2} \phi=4 \pi G \mu
$$

Now let us find the component of five-potential whose source is temperature. In this case the current five-vector would be

$$
J^{\mu}:=\left(\theta k_{B} \sigma_{0} / c, c \rho, \mathbf{J}\right)
$$

where $\theta$ is temperature density per unit volume and $k_{B}$ Boltzmann constant. To find the corresponding component of five-potential we must follow the above-mentioned recipe, except that now we do not know the corresponding potential for temperature and it is in fact our goal to find it. Let us therefore suppose that the corresponding potential is denoted by $\xi$. Applying our recipe and dimensional analysis simultaneously, we must have

$$
\left[\frac{\xi c}{k_{B} \sigma_{0}}\right]=\frac{\text { volt }}{[c]}
$$

immediately it would follow that

$$
[\xi]=\text { Joules } / \text { Kelvin }
$$


which is the same dimensions as that of entropy, therefore the corresponding potential for temperature is entropy. The field equation

$$
\square A^{\mu}=-\mu_{0} J^{\mu}
$$

would now yield the proposed equation (2).

\subsection{Law of Conservation of Informedness}

$$
\frac{\partial \iota(\mathbf{x}, t)}{\partial t}=-\nabla \cdot(\iota \mathbf{v})
$$

Although similar in name, this conservation is a classical law and has nothing to do with the famous statement from quantum mechanics that the quantum-mechanical wave function is the carrier of information, in other words, complete information about a system is encoded in its wave function up to when the wave function collapses. That quantum mechanical statement is theoretically vague for it is based on the informational interpretation of quantum mechanics, so it is dependent upon interpretation in a theory whose advocates abhor interpretation in favour of although fictitious - mathematical transparency and well-definedness, so the dogma of conventional quantum mechanics calls for some equation, but is unable to provide any such equation and the statement by their standards remains in the level of an statement, not a principle or law.

\subsection{So what is heat, exactly?}

We are now able -unlike the established theory- to understand heat in an exact and quantitative manner: Heat is the gradient of the entropy field, as defined by equation (2), viz.

$$
\mathbf{C}=-\nabla S
$$

where $\mathbf{C}$ stands for calor $^{9}$.

\section{Regarding Blackhole Thermodynamics, Part I}

Our proposed fundamental law (2) suggests that temperature (or informedness) is not a statistical quantity but a fundamental property of elementary particles, very much like mass or electric charge. If (2) is a true law of nature, then entropy is re-defined without any reference to microstates; the only example of such encounter we already know in theoretical physics is the Bekenstein-Hawking entropy of a Schwarzschild blackhole[10],

$$
S_{\mathrm{BH}}=\frac{k_{B} A}{4 l_{P}^{2}}
$$

\footnotetext{
${ }^{9}$ Latin for heat.
} 
where $A$ is the area of blackhole's event-horizon and $l_{P}=\sqrt{\hbar G / c^{3}}$ is the Planck length.

With (2) in mind in one hand, and a statistical definition of entropy $S=k_{B} \log W$ on the other hand, we are faced with a dichotomy:

A) According to the statistical definition of entropy, the entropy of a Schwarzschild blackhole (4) must come from an ensemble of microstates, whereas

B) Since a Schwarzschild blackhole is formally no different from an uncharged spin-0 elementary particle ${ }^{10}$, according to (2) a (Schwarzschild) blackhole does fundamentally possess temperature, rendering any talk of microstates redundant, if not meaningless.

The only simple and rather-satisfactory treatment along $\mathbf{A}$ is the work of Rovelli[12] with the assumption of the quantisation of area, '[...] based on the idea that the entropy of the hole originates from the microstates of the horizon that correspond to a given macroscopic configuration', Rovelli took it that 'in a thermal context, the Schwarzschild metric represents the coarse-grained description of a microscopically fluctuating geometry.' There is a serious logical objection to the work of Rovelli, which finally leads to an infinite regress or a contradiction:

Since a Schwarzschild blackhole and an uncharged spin-0 elementary particle are formally the same, The mere acceptance of this statement is a contradiction with the statistical definition of temperature: it means that one has accepted that a single particle possesses temperature! To see how this leads to an infinite regress let us review what has happened to this position in the argument: One came defending the position that temperature is a property of many particles, then admitted it can be attributed to a single particle only to defer its cause to 'microstates of geometry'; but logically continuing, those microstates themselves must possess temperature coming from microstates of geometry, of geometry ad infinitum, unless there is a lower limit to the geometry of microstates of that elementary particle, for instance Higgs boson, which has to be the Schwarzschild radius of the particle

$$
r_{s, \mathrm{H}^{0}}=\frac{2 G M_{\mathrm{H}^{0}}}{c^{2}} \approx 3.2 \times 10^{-52} \text { meters } \approx 8.3 \times 10^{-23} l_{P}
$$

which is astronomically smaller than the Planck length and no conventional theory of quantum gravity can ever hope to probe that scale, given that theories such as the loop quantum gravity are promised to work in orders of $l_{P}$, therefore this alternative has led to a contradiction too.

\section{An argumentum ad absurdum}

As we argued in section (2) the current statistical definition of entropy is logically flawed. To make the problem more clear we now prove that with regard to a statistical treatment of emergence, both mass and temperature are the same; i.e. either they are both emergent and non-attributable to

\footnotetext{
${ }^{10}$ To ensure there are no misunderstandings, we emphasize that a Schwarzschild blackhole and an elmentary particle are formally the same, i.e. only the numerical values of their masses differ, and both are in the realm of quantum gravity.
} 
a single particle, or they are. In other words, saying that temperature cannot be attributed to a single (elementary) particle is as absurd as saying that mass cannot be attributed to a single (elementary) particle!

\section{1 'Statistical definition' of gravitational poten- tial and mass}

The fundamental equation proposed in section 4 is based on the analogy that entropy is playing the same role for temperature that gravitational potential plays for mass. Suspending our logical judgement for a moment and looking at this analogy the other way around, if the potential function of temperature can be defined by $S=k_{B} \log W$, we conclude that we can define the potential function of mass (i.e. the gravitational potential $\phi$ ) by

$$
\phi:=c^{2} \log W_{g}\left(E_{g}\right)
$$

where $c$ is the speed of light in vacuum, and $W_{g}\left(E_{g}\right)$ is the number of 'microstates' that this particle has a fixed rest-energy $E_{g}$.

Continuing with the analogy, we can 'define" ${ }^{, 11}$ the rest-mass ${ }^{12}$ of this particle $m$ to be

$$
\frac{1}{m}:=\frac{\partial \phi}{\partial E_{g}}
$$

\subsubsection{Gravitational Capacity (Massic/Specific energy)}

Similar to the definition of Heat capacity, if we add volume-dependence of microstates to the 'statistical definition' of $\phi$, viz.

$$
\phi:=c^{2} \log W_{g}\left(E_{g}, V\right)
$$

we can even statistically define the specific energy or massic energy, which we call Gravitational Capacity, by

$$
e:=\frac{\partial E_{g}}{\partial m}
$$

\subsubsection{Gravitational Pressure}

$$
p_{g}:=m \frac{\partial \phi}{\partial V} .
$$

\subsubsection{First 'law' of Gravitodynamics}

Similar to the first law of thermodynamics, as a result of above definitions, the following relation holds

$$
d E=m d \phi-p_{g} d V
$$

We may now express the arrow of time, by

$$
d \phi \geq 0
$$

\footnotetext{
${ }^{11}$ Mind the quotation marks; we do not regard this as a definiton because it is circular. We are doing this only to illuminate our point.

${ }^{12}$ Erik Verlinde[13] would probably consider this as 'an explanation' of inertia.
} 
Once the above definitions of gravitational potential and rest-mass are given, it is not hard to construct the rest of the theory similar to statistical mechanics. Therefore we do not indulge in details of derivation here and only mention the final results. The reader can follow the details in any proper statistical mechanics book like [2][3][4].

\subsection{The canonical ensemble}

$$
p(n)=\frac{e^{-E_{n} / m c^{2}}}{\sum_{m} e^{-E_{m} / m c^{2}}}
$$

\subsection{Equipartition of energy}

In the canonical ensemble, the system is in equilibrium with an infinite mass reservoir at $m$. The probability of each state in phase space is given by

$$
e^{-\frac{\epsilon_{i}}{m c^{2}}}
$$

times a normalization factor $\mathcal{N}_{g}$, which is chosen so that the probabilities sum to one

$$
\mathcal{N}_{g} \int e^{-H(p, q) / m c^{2}} d \Gamma=1
$$

After integration by parts for a phase-space variable $x_{k}$ and the intermediate steps, we arrive at

$$
\left\langle x_{i} \frac{\partial H}{\partial x_{j}}\right\rangle=\delta_{i j} m c^{2}
$$

\section{Mass-Charge correspondence}

\subsection{Introduction}

Continuing with our maxim that a blackhole and an elementary particle are formally the same, we need to justify the attribution of Schwarzschild radius to elementary particles, e.g. electron. To this end we posit that $E=m c^{2}$ is the gravitational energy of a particle. To find the gravitational energy of a particle we need a preliminary discussion, which will also give us a rest-energy for charged particles and consequently predicts the existence of a massless charged particle.

\subsection{The correspondence}

In the context of classical physics, a fundamental difference exists between mass and electric charge: Mass appears in the most fundamental law of classical physics, i.e. Newton's Second Law and from there it makes its influence passed to Electromagnetism, Relativity and Quantum Theory. The fact that in the classical law of motion, i.e. Newton's Second Law only mass exists accounts for Einstein's (Weak) Equivalence Principle, viz.

$$
m \mathbf{g}=m \mathbf{a}
$$


allows for the cancellation of $m$ and identification of the gravitational field with acceleration, which in turn makes possible the identification of metric tensor components with the gravitational potential through the comparison of (12) and the geodesic equation.

Yet as we think deeply no fundamental reason exists as to why we should differentiate these two concepts; It might be said that mass is a criterion for physical existence of a particle while charge is not, but then no massless particle should exist, which we know is in stark contrast with empirical evidences. Therefore to treat electric charge and mass equally, we must find a conversion factor between the two. We do this by a simple dimensional analysis, using the fundamental constants of an illusive theory of gravitoelectromagnetism, i.e. $\epsilon_{0}, \mu_{0}$ and $G$ :

$$
\text { [electric charge }]\left[\epsilon_{0}\right]^{\alpha}\left[\mu_{0}\right]^{\beta}[G]^{\gamma}=[\text { mass }]
$$

Note that to follow our initial goal of treating mass and charge completely equal, we must take the power of electric charge to be the same as of mass. We therefore have

$$
\alpha=\frac{-1}{2}, \beta=0, \gamma=\frac{-1}{2}
$$

up to a constant which we choose to be $1 / \sqrt{16 \pi}$ to make the Coulomb constant appear in the conversion factor.

\subsection{Implications}

\subsubsection{Charge-Energy equivalence}

A logical conclusion is charge-energy equivalence, i.e. energy possessed by merely the electrically charged aspect of a particle is given by,

$$
E=\frac{q c^{2}}{\sqrt{16 \pi \epsilon_{0} G}}
$$

\subsubsection{Existence of Massless Charged particles}

By the previous considerations we propose the existence of a massless particle with electric charge $e^{13} e$. The energy for creation/annihilation of such a particle would be

$$
E=\frac{q c^{2}}{\sqrt{16 \pi \epsilon_{0} G}} \approx 8.35 \times 10^{7} \text { Joules }
$$

\subsubsection{Reissner-Nordström radius from posited Electrostatic energy}

Suppose we take a simple model of an elementary charged particle in which all of its charge $q$ is uniformly distributed on the surface of a sphere of radius $r$, if the charge is standing still, there is no magnetic field, and

\footnotetext{
${ }^{13} \mathrm{We}$ do this since, according to our current understanding, the quanta of electric charge is $e$ and quarks are not ever observed in isolation (the quark confinement); but according to $\mathrm{QCD}$, this number should be divided by three, as quarks possess charges which are integer multiples of $\frac{1}{3} e$.
} 
the energy per unit volume is proportional to the square of the electric field. Therefore the energy of electron's electric field is

$$
E=\frac{1}{2} \frac{q^{2}}{4 \pi \epsilon_{0}} \frac{1}{r}
$$

Now, we posit that $q c^{2} / \sqrt{16 \pi \epsilon_{0} G}$ is the electrostatic energy of this modeled charged particle. Therefore equating (13) and (14) yields

$$
r=r_{\mathrm{RN}}=\frac{q}{c^{2}} \sqrt{\frac{G}{4 \pi \epsilon_{0}}}
$$

which is the characteristic length appearing in the Reissner-Nordström metric solution of Einstein-Maxwell field equations.

\subsubsection{Schwarzschild radius from posited Gravitational en- ergy}

To find the gravitational energy of a spherical mass $m$ we follow an indirect path, which will justify the mass-charge correspondence. According to our charge-mass correspondence, in (14) we put

$$
q \equiv m \sqrt{16 \pi \epsilon_{0} G}
$$

to get

$$
E=2 m^{2} G \frac{1}{r}
$$

now, as we have positted that $E=m c^{2}$ is the gravitational energy of this modeled particle, we equate (8) with $m c^{2}$, which yields

$$
r_{s}=\frac{2 G m}{c^{2}}
$$

which is the Schwarzschild radius.

It must be evident by now that taking a blackhole and an elementary particle formally the same is completely justified. Therefore we have finally proved that position $\mathbf{A}$ in the argument of previous section is untenable.

\subsection{A closer look at the Reissner-Nordström met- ric}

Now that we have shown that treating mass and charge on completely equal grounds is justified by leading to correct radii, if we look at the Reissner-Nordström metric solution of Einstein-Maxwell equations,

$$
d s^{2}=\left(1-\frac{r_{s}}{r}+\frac{r_{\mathrm{RN}}^{2}}{r^{2}}\right) c^{2} d t^{2}-\left(1-\frac{r_{s}}{r}+\frac{r_{\mathrm{RN}}^{2}}{r^{2}}\right)^{-1} d r^{2}-r^{2} d \Omega^{2}
$$

we realise that this metric does not respect the correspondence: the powers of mass and charge are not equal. This was not entirely unexpected since Einstein-Maxwell equations are not a true unification of gravity and 
electromagnetism. Following our correspondence, we propose the following (static) metric for a charged, non-rotating, spherically symmetric body of mass $M$ and charge $q$,

$$
d s^{2}=\left(1-\frac{r_{s}}{r}-\frac{r_{\mathrm{RN}}}{r}\right) c^{2} d t^{2}-\left(1-\frac{r_{s}}{r}-\frac{r_{\mathrm{RN}}}{r}\right)^{-1} d r^{2}-r^{2} d \Omega^{2}
$$

which completely respects mass-charge similarity, but ought to represent the gravitoelectric field of the body, not solely its gravitational field. To see this point better, note that according to mass-charge correspondence, a particle in the (static) gravitoelectric field experiences a total potential of

$$
\phi+\sqrt{16 \pi \epsilon_{0} G} \varphi
$$

where $\phi$ is the gravitostatic potential (Newtonian gravitational potential) and $\varphi$ the electrostatic potential (Coulomb potential).

Therefore where using the geodesic equation

$$
\frac{d^{2} x^{\mu}}{d s^{2}}+\Gamma_{\rho \sigma}^{\mu} \frac{d x^{\rho}}{d s} \frac{d x^{\sigma}}{d s}=0
$$

by passing to the Newtonian limit, we make the identification

$$
g_{00}=-(1+2 \phi),
$$

(16) suggests that we must instead make the following identification to fully respect mass-charge similarity

$$
g_{00}=-\left(1+2 \phi+2 \sqrt{16 \pi \epsilon_{0} G} \varphi\right) .
$$

Therefore according to (17) the metric tensor represents both gravitostatic and electrostatic fields, not solely the gravitational field; hence the name gravitoelectric.

\subsubsection{Empirical Preference}

A question naturally arises regarding the discussion about (16), that which one is the correct metric, correctly representing the geometry of space-time around a static spherically symmetric charged blackhole? As is usual in physics, the last word can only be said when empirical investigations rule out one of the possibilities.

\section{Regarding Blackhole Thermodynamics, Part II}

\subsection{Thermal radius of a spherically symmetric entity}

Following a similar reasoning to that of previous section, positing that $E=2 k_{B} T$ is the thermal energy of a particle we arrive at the thermal radius of an entity (particle or blackhole) given by

$$
r_{\theta}=\frac{4 G k_{B} T}{c^{4}}
$$


14 If we now let

$$
r_{\theta} \equiv \frac{\hbar}{2 \pi M c}
$$

(Reduced Compton wavelength divided by $2 \pi$, which we call DoublyReduced Compton Wavelength) we arrive at

$$
T=\frac{\hbar c^{3}}{8 \pi G k_{B} M}
$$

which is the Hawking temperature[10].

\subsection{Inclusion of blackhole's Temperature in the metric}

Here again we face the same dichotomy faced in section 7.4, namely whether we should take a Reissner-Nordström-like approach, which is the result of solving Einstein field equations and (2); or, we should respect the mass-charge correspondence, which by now is promoted to mass-chargetemperature correspondence. As we said there, we shall remain open to both possibilities until empirical evidence rules out one of them.

Therefore for a static spherically symmetric uncharged blackhole with mass $M$ and temperature $T$, we can follow two possible approaches:

\subsubsection{Reissner-Nordström-like approach}

$$
d s^{2}=\left(1-\frac{r_{s}}{r}+\frac{r_{\theta}^{2}}{r^{2}}\right) c^{2} d t^{2}-\left(1-\frac{r_{s}}{r}+\frac{r_{\theta}^{2}}{r^{2}}\right)^{-1} d r^{2}-r^{2} d \Omega^{2}
$$

\subsubsection{Mass-temperature correspondence approach}

$$
d s^{2}=\left(1-\frac{r_{s}}{r}-\frac{r_{\theta}}{r}\right) c^{2} d t^{2}-\left(1-\frac{r_{s}}{r}-\frac{r_{\theta}}{r}\right)^{-1} d r^{2}-r^{2} d \Omega^{2}
$$

\section{Objections and Replies}

\subsection{Temperature à la Landau-Lifshitz}

'When discussing the "definition of temperature", the author seems to have overlooked an important issue. Namely, when starting from the number $W(E)$ of states accessible to an isolated system with energy $E$, and introducing the entropy as the logarithm of that number without invoking Boltzmann's constant, one may define a "natural temperature" according to Eq. (1) in this manuscript. With the entropy now being dimensionless, that natural temperature bears the same unit as energy. What is more, it

${ }^{14}$ If we allow ourselves to effectively (not ontologically original) talk about the microstates of blackholes, as the thermal radius of e.g. Sun is

$$
r_{\theta}, \text { Sun }=\frac{4 G k_{B}(5778 \text { kelvins })}{c^{4}} \approx 2.6 \times 10^{-63} \text { meters },
$$

it is conceivable that such 'microstates of geometry' occur at scales comparable to $r_{\theta}$, Sun, which is much smaller than $l_{P}$. 
is not difficult to show that the statistically defined natural temperature shares essential properties of the phenomenologically known temperature: Two systems in thermal contact acquire the same temperature, and heat always flows from the system with the higher temperature to the one with the lower temperature. Thus, it is clear right from the outset that the above defined natural temperature is monotonically related to the preexisting phenomenological temperature, measured in Kelvin. What is not trivial at all, however, is the that the two temperature scales, introduced apparently independently from each other, actually agree up to a constant of of proportionality, which is Boltzmann's constant. Thus, if one wishes that the statistical temperature even agrees with the phenomenological one, one merely needs to rescale the entropy by multiplying the logarithm of $W(E)$ by the Boltzmann constant. This recognition is one of the key insights of statistical physics, which is why it is engraved on Boltzmann's tombstone. The author's claim that there is an "impossibility of emergence of temperature within current physics" is, therefore, based on an insufficient understanding of both thermodynamics and statistical physics. ${ }^{15}$

\subsubsection{Reply}

One must be bound by a certain definition and changing the definition at the end is absurd (as does the author of this objection in the end by "rescaling" temperature). A "natural temperature" whose unit is energy is not temperature anymore, it is energy! as we know that the unit of a physical quantity is a necessary part of its definiens.

This objection can be dressed differently by saying that we take $k_{B}=1$, which is essentially the lesson of relativity first done to $c$. It means that

$$
1 \text { Kelvin }=1.380649 \times 10^{-23} \text { Joules, }
$$

Assuming for the moment that "natural temperature" is the correct definition of temperature, with same units as energy, we again refer to

$$
\frac{1}{T}:=\frac{\partial S}{\partial E}
$$

We see that we are now using energy to define a quantity whose units is same as energy by assumption! Therefore this is again a logical circle! This 'objection', therefore, is based on an old dogma of Academia, that something which is not mentioned by the author is unknown to the author. This approach to 'defining' temperature was known to the author from the beginning (as should be, for no scientist begins to criticise established theories without having a proper understanding and reading of the literature), but he did not think that such superficiality were at the work of a so-called scientific journal. By the way, I cannot see the relevance of any objection to my work to Boltzmann's tombstone $(!)^{16}$.

\footnotetext{
${ }^{15}$ by Zeitschrift für Naturforschung $A$.

${ }^{16}$ Other than the implication that I have committed a sacrilegious act of blasphemy, perhaps by treading on Boltzmann's tombstone!
} 


\subsection{Theoretical physics methodology à la pop- science}

'The author is claiming that entropy is playing the same role for temperature as the gravitational potential plays for the mass. However, this claim is not systematically derived, but merely proposed on the grounds of handwaving arguments. In sharp contrast, theoretical physics is not about making unfounded propositions, but about explaining experimental observations. This brings me to my major item of criticism: The present manuscript does not explain anything, and remains entirely unrelated to any observable physics. In particular, no observable consequences are deduced from the propositions which would allow one to either confirm or falsify the present speculations, or would yield some new insight into physics. The authors has not even bothered to discuss the alleged main findings of this work in a decent summary. ${ }^{17}$

\subsubsection{Reply to: 'not systematically derived'}

The esteemed reviewer sounds to be totally alien to the methodology of physics and is only repeating some old empiricist dogma found in popscience books! Anyone who has spent at least one hour thinking about how physics works, knows that nothing in physics can be 'derived', let alone 'systematically' derived, for systematically means that a closed system of axioms is at work. So in a system whose one of foundational assumptions is the emergence of temperature, of course, it cannot be 'systematically derived' that temperature is not emergent! That would be a sheer contradiction! The only thing that a theorist can do is to look for motivations in established theories and try to push the foundations using those motivations/justifications.

If the reviewer read manuscript with a speck of good will ${ }^{18}$ he would immediately see that this paper is going deep into the very foundations of thermodynamics and is a proposing a whole new set of axioms.

\subsubsection{Reply to: 'physics is about explaining experimental observations'}

This again is hardly better than being spoon-fed by a pop-science book, without ever having done any serious theoretical physics. No, theoretical physics is not about explaining experimental observations. Do I need to repeat the old examples of Lagrange, Hamilton, Einstein, Dirac, etc.? Empirical judgement is but one among many judgements that are available in theoretical physics. The Ptolemic (geocentric) system also 'explained' all experimental observations in its time. So your journal could have easily rejected the work of Copernicus and Kepler based on this objection in a time that Galileo's telescope was not yet available.

A modified newtonian potential was available before Einstein's General

\footnotetext{
${ }^{17}$ by Zeitschrift für Naturforschung A.

${ }^{18}$ There is a strong possibility that they did not even read it but such assumption would be in contrast to how scientific conduct is supposed to work.
} 
theory of Relativity that could well 'explain' Perihelion precession of Mercury. Why not accept that?

What was the main motivation for Dirac in his equation? Could not Pauli's equation 'explain' observations of spin? Inconsistency (arguments from harmony) has been a much more powerful criterion for judging theories and seeking better ones in modern times.

\subsubsection{Reply to: 'does not explain anything'}

If one has any sane understanding of the world 'explain', well the proposal 'explain's many things:

1. What exactly is heat?

2. What exactly is entropy?

3. What exactly is temperature?

4. The well-known contradiction between heat conduction and locality (Special Relativity)

5. The old question of the temperature of a moving body[14], i.e.

$$
T \rightarrow \gamma T
$$

inter alia.

\subsubsection{Reply to: 'no observable consequences'}

It seems that you have not paid enough attention to equation (2): It is a wave equation for entropy! So it is evidently predicting existence of entropic waves.

But to be not as superficial as the reviewer, there is a point here, which ultimately leads to the question

Is the observation of gravitational waves an evidence for $\square \phi=-4 \pi G \mu$, or is it an evidence for geometrisation of forces -regardless of any particular force? To see that this is the ultimate question that should be asked in this regard, adopting the position of a historical observer is useful: Suppose somebody in 18th century arrives at $\square \phi=-4 \pi G \mu$ and says that there must exist gravitational waves, but he cannot propose any practical method to observe such waves and academia would easily neglect his work $^{19}$; what Einstein does which makes him famous, is to say that length contraction is the evidence for such gravitational waves, something which our 18th-century physicist could not do, although we will argue somewhere else that the observation of gravitational waves is an evidence for geometrisation of all forces, not only gravitational waves, and these two are different, two questions should be asked:

- How do you observe such entropic waves?, to which I answer 'I do not know'.

\footnotetext{
${ }^{19}$ In fact this is almost what happened to Gunnar Nordström, who proposed $\square \phi=-4 \pi G \mu$ shortly after Special Relativity[15][16].
} 
- Would it be fair to neglect your work just like Nordström's[16], in the hope of the rise of somebody in future who can tell us how to observe these entropic waves?, to which I would immediately answer 'absolutely not', because the situation is totally different from $\square \phi=-4 \pi G \mu$. Neglecting my work is just like neglecting Newtonian gravity in the hope that somebody in future would 'explain' the field nature of gravity. In the case of Nordström, people at least accepted the Poisson equation for gravity. Here, with entropy, the whole concept of a field of heat whose potential function is entropy is utterly radical and unacceptable to the establishment. So we are essentially trying to do for heat what Newton did for gravity, not what Einstein did. Expecting to make the (analogous) progress of both Newton and Einstein simultaneously is just unfair to the historical evolution of scientific ideas. In other words, for Einstein to propose how to observe gravitational waves, there must already exist beforehand a Poisson equation and a potential function. ${ }^{20}$

\section{A History of evolution of author's ideas which led to field approach to Entropy}

This work can be considered as a spin-off from a grand programme: Unification of forces à l'Einstein

\section{References}

[1] Gratton, C.(2009). Infinite Regress Arguments. Springer Netherlands. p. 296. ISBN 978-90-481-3340-6.

[2] Reif, F. (1965). Fundamentals of Statistical and Thermal Physics. McGraw-Hill. p. 99. ISBN 9780070518001.

[3] Chandler, David (1987). Introduction to Modern Statistical Mechanics. Oxford University Press. p. 61. ISBN 0-19-504277-8.

[4] Kittel, Charles; and Kroemer, Herbert (1980) New York: W.H. Freeman. p. 41. ISBN 0-7167-1088-9

[5] http://blogs.nature.com/news/2012/08/hot-stuff-cern-physicistscreate-record-breaking-subatomic-soup.html

[6] Eckert, E. R. G.; Drake, R. M. (1972). Analysis of Heat and Mass Transfer. Tokyo: McGraw-Hill, Kogakusha.

[7] Cattaneo, C. R. (1958). "Sur une forme de l'équation de la chaleur éliminant le paradoxe d'une propagation instantanée". Comptes Rendus. 247 (4): 431.

[8] Vernotte, P. (1958). "Les paradoxes de la theorie continue de l'équation de la chaleur". Comptes Rendus. 246 (22): 3154.

\footnotetext{
${ }^{20}$ Thinking by historical analogy, we must ask How could have Nordström defended $\square \phi=$ $-4 \pi G \mu$ empirically, without having gravity geometrised? This question is the main question around this issue that the author has been dealing with.
} 
[9] Chester, M. (1963). "Second sound in solids". Physical Review. 131 (15): 2013-2015. Bibcode:1963PhRv..131.2013C. doi:10.1103/PhysRev.131.2013.

[10] Hawking, S. W (1975). "Particle creation by black holes". Communications in Mathematical Physics. 43 (3): 199-220. Bibcode:1975CMaPh..43..199H. doi:10.1007/BF02345020

[11] Bekenstein, Jacob D. (April 1973). "Black holes and entropy'. Physical Review D. 7 (8): 2333-2346. Bibcode:1973PhRvD...7.2333B. doi:10.1103/PhysRevD.7.2333.

[12] Rovelli, Carlo (1996). "Black Hole Entropy from Loop Quantum Gravity". Physical Review Letters. 77 (16): 3288-3291. arXiv:gr-qc/9603063. Bibcode:1996PhRvL..77.3288R. doi:10.1103/PhysRevLett.77.3288

[13] Verlinde, E.P. (2011). "On the Origin of Gravity and the Laws of Newton". JHEP. 2011 (4): 29. arXiv:1001.0785. Bibcode:2011JHEP...04..029V. doi:10.1007/JHEP04(2011)029. S2CID 3597565

[14] Farías, C., Pinto, V.A. \& Moya, P.S. "What is the temperature of a moving body?". Sci Rep 7, 17657 (2017). https://doi.org/10.1038/s41598-017-17526-4

[15] Deruelle, N. "Nordström's scalar theory of gravity and the equivalence principle". Gen Relativ Gravit 43, 3337-3354 (2011). https://doi.org/10.1007/s10714-011-1247-x

[16] Ravndal, F. (2004). "Scalar Gravitation and Extra Dimensions". arXiv:gr-qc/0405030v1 